\title{
Evaluating performance of supply chain based on network data envelopment analysis
}

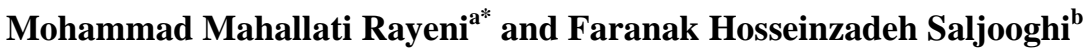

${ }^{a}$ Faculty of Bahonar, Technical and Vocational University, Zahedan, Iran

${ }^{b}$ Department of Mathematics, University of Sistan and Baluchestan, Zahedan, Iran

\section{H R O N I C L E}

\section{Article history:}

Received June 12, 2012

Received in revised format

28 October 2012

Accepted 30 October 2012

Available online

November 32012

Keywords:

Supply chain

Data envelopment analysis

Efficiency

Network model

Cooperative and non-cooperative

models \begin{abstract}
A B S T R A C T
Supply chain is one of the most important parts of industrial and economic problems. Measuring relative performance of supply chain is often tedious task since there is a need to deal with the multiple performance measures associated with members of supply chain, which integrate and coordinate the performance overall production system. Traditional approaches for measuring relative efficiency of supply chain cannot be applied directly for evaluating the relative efficiency of supply chains. This is because some measures linked to supply chain members cannot be simply classified as "outputs" or "inputs" of the supply chain. We present different models, which directly evaluate the performance of the supply chain as well as its members, while considering the relationship among the members. The modeling processes are based on the concept of non-cooperative and cooperative games. In this paper, we propose network data envelopment analysis for measuring relative performance of supply chain, which considers the system by distinct processes or stages, each one with its own inputs and outputs and with intermediate flows among the stages. The proposed method reduces computations to determine the relative efficiency of overall supply chain and efficiency of each the supply chain members.
\end{abstract}

\section{Introduction}

A supply chain (SC) includes all components involved, directly or indirectly in accomplishing a customer's needs. SC not only includes the manufacturer and suppliers, but also other particles such as transportations, retailers, warehouses, and customers. A SC is a cooperation of independent business components such as supplier, distribution processes and manufacturing, which perform the critical functions to fulfill process. SC management (SCM) is a set of particles utilized to integrate suppliers, warehouses, manufacturers specifically and stores so that merchandise is performed and distributed more specifically to the right locations, on time with a purpose of minimizing total expenditures while meeting service level requirements. When we make an assessment on the efficiency of a SCM, we require to measure the performance of each individual SC components by

\footnotetext{
*Corresponding author

E-mail addresses: m_mohallati@yahoo.com (M. Mahallati) 
considering all possible relationships among various components and SCM strategies cannot be determined, separately. All components of SCM are normally impacted by other components, at the same time. SC strategies also should be aligned with other organizational objectives such as maximizing market share or improving profitability or minimizing expenditures. However, shortage of appropriate performance measurement systems has been an obstacle to an effective SCM implementation.

Data envelopment analysis (DEA) is a typical linear programming based framework for measuring the relative efficiency of each member of a set of organizational units. The units, called decisionmaking units (DMUs), consume various levels of each specified input and produce various levels of each specified output. Standard DEA models cannot be implemented directly to measure the performance of a supply chain and its members, because of the existence of the intermediate measures connecting those members. This observation holds under any circumstances where DMUs contain multi-stage processes. An important area of development has been considered to areas where in DMUs represent multi-stage or network processes.

So far, the value-added processes or systems have been considered as a "black-box" but each process or system can incorporate various sub-processes. For instance, if the process is to build a car, then important sub-processes include assembling parts and painting. If we make an assessment on the efficiency of a SCM, then we require to measure the performance of each individual SCM components. While there are studies on SCM performance using DEA, the focus is on a single member of the SCM. Within the context of DEA, there are a number of techniques to be implemented in SCM efficiency evaluation. Seiford and Zhu (1999) and Chen and Zhu (2004) provided two approaches in modeling efficiency as a two-stage process. Cook et al. (2010) reviews some two-stage models and established relationships among various approaches. Färe and Grosskopf (2000) developed the network DEA approach to model general multi-stage processes with intermediate inputs and outputs. Golany et al. (2006) provided an efficiency measurement framework for systems composed of two subsystems arranged in series that simultaneously compute the efficiency of the aggregate system and each subsystem. Kao (2009) considered two parallel and series structures for internal parts of DMU in order to investigation network model. Liang et al. (2006) developed two classes of DEA-based models for supply chain efficiency evaluation based on non-cooperative model.

In this study, we examine efficiency of SCM based on network DEA in two methods. In first method, we measure overall efficiency of SCM as the product of the efficiencies of members. The second method computes overall efficiency as the weighted sum of the efficiencies of members. Then, we compare these two methods. The rest of the paper is organized as follows. Section 2 reviews the conventional DEA. Section 3 is allocated to DEA models are developed for supply chain, under the assumption that the relationship between the members is treated as leader-follower and then in section 4 we discuss cooperative models as network DEA.

\section{Data envelopment analysis (DEA)}

DEA has been proven a useful technique for evaluating relative performance of homogeneous decision-making units (DMUs) in a multiple-input multiple-output setting. Traditional DEA estimates the efficiency index by measuring the ratio of weighted outputs to weighted inputs, and the input and output weights are decided based on the best interests of the DMUs. Consider $n$ decision making units (DMUs) to be evaluated, each indexed by $j(j=1,2, \ldots, n) ; D M U_{j}(j=1,2, \ldots, n)$ consume $m$ inputs $\left(x_{i}: i=1,2, \ldots, m\right)$ to produce $s$ outputs $\left(y_{r}: r=1,2, \ldots, s\right)$. The CCR input oriented (CCR-I) model evaluates the efficiency of $D M U_{k}$, DMU under consideration, by solving the following linear program: 
$\max \sum_{r=1}^{s} u_{r} y_{r k}$

subject to

$$
\begin{array}{ll}
\sum_{r=1}^{s} u_{r} y_{r j}-\sum_{i=1}^{m} v_{i} x_{i j} \leq 0 & j=1, \cdots, n \\
\sum_{i=1}^{m} v_{i} x_{i k}=0 & \\
u_{r} \geq 0, v_{i} \geq 0 & r=1, \cdots, s \quad \mathrm{i}=1, \cdots, m
\end{array}
$$

Duality of model (1) is as follow:

$\theta_{k}^{*}=\min \quad \theta_{k}$

subject to

$\sum_{j=1}^{n} \lambda_{j} x_{i j} \leq \theta x_{i k}$

$$
i=1, \cdots, m
$$

$$
\sum_{j=1}^{n} \lambda_{j} y_{r j} \geq y_{r k}
$$

Definition 1. $D M U_{k}$ is efficient if $\theta_{k}^{*}=1$, Otherwise, $D M U_{k}$ is inefficient.

\section{Supply chain in DEA}

SCM has been proven to be effective tool to provide prompt and reliable delivery of high-quality products and services at the least cost. To achieve this, performance evaluation of entire supply chain is extremely important, since it means utilizing the combined resources of the supply chain in the most efficient possible way to incorporate market wining and cost-effective products and services. Two hurdles are present in measuring the performance of value chains. One is the existence of multiple measures, which characterize the performance of each member in a supply chain. The other is the conflicts between SC members with respect to specific measures. SC systems can be viewed as an integrated input-output system where each supply chain member implements inputs to produce.

Suppose we have $h$ observations associated with each supply chain member, i.e., we have observed input values of $x_{i j}^{k} \quad(i=1,2, \ldots, m)$ and output values $y_{r j}^{k}(r=1,2, \ldots, s)$, also $z_{d j}^{(k, t)}(d=$ $1, \ldots, D)$ is $d^{\text {th }}$ intermediate output from the member $k$ to member $t$ (also represents an input to member $t$ ) where $j=1, \ldots, n$. Note that only intermediate outputs are defined, since each such output also represents an input to an associated supply chain member. The efficiency of $\mathrm{k}^{\text {th }}$ supply chain member $t$ can be measured by the following DEA model - input oriented CCR model.

$$
\theta^{*}=\min \frac{\sum_{t=1}^{h} w_{t} \theta_{t}}{\sum_{t=1}^{h} \theta_{t}}
$$

subject to 
$\sum_{j=1}^{n} \lambda_{t j} x_{i j}^{t} \leq \theta_{t} x_{i k}^{t}$

$\sum_{j=1}^{\mathrm{n}} \lambda_{t j} \mathrm{y}_{\mathrm{rj}}^{\mathrm{t}} \geq \mathrm{y}_{\mathrm{rk}}^{\mathrm{t}}$

$\sum_{j=1}^{n} \lambda_{t j} z_{d j}^{(t, k)} \geq z_{d k}^{(t, k)}$

$\lambda_{\mathrm{tj}} \geq 0$

$i=1, \ldots, m, \mathrm{t}=1, \ldots, \mathrm{h}$

$$
\mathrm{r}=1, \ldots, \mathrm{s}, \mathrm{t}=1, \ldots, \mathrm{h}
$$

$\mathrm{d}=1, \ldots, \mathrm{D}, \mathrm{t}=1, \ldots, \mathrm{h}$

$j=1, \ldots, n \quad f, k \in(t=1, \ldots, h)$

where $\theta_{t}$ is efficiency of member $t$ and $w_{t}$ be the user-specified weights reflecting the preference over supply chain member's performance.

Definition 2 (Efficient Supply Chain) A supply chain is efficient if $\theta^{*}=1$, where $\theta^{*}$ is the optimal value to model (3).

\section{Cooperative and non-cooperative approaches}

In this section, we present several models, which directly evaluate the performance of the supply chain as well as its members, while considering the relationship between the members. The modeling processes are based on the idea of non-cooperative and cooperative games.

\subsection{Non-cooperative models}

Suppose there are $n$ similar supply chains. Consider a buyer-seller supply chain as described in Fig. 1 , where for $j=1, \ldots, n, X^{A}=\left(x_{i j}^{A} ; i=1,2, \ldots, m\right)$ is the input vector of the seller, and $Y^{A}=$ $\left(y_{r} ; r=1,2, \ldots, s\right)$ is the seller's output vector, $Y^{A}$ is also an input vector of the buyer. The buyer also has an input vector $X^{B}=\left(x_{i j}^{B} ; i=1,2, \ldots, m\right)$ and the output vector for the buyer is $Y^{B}=$ $\left(y_{i r}^{B} ; r=1,2, \ldots, s\right)$.

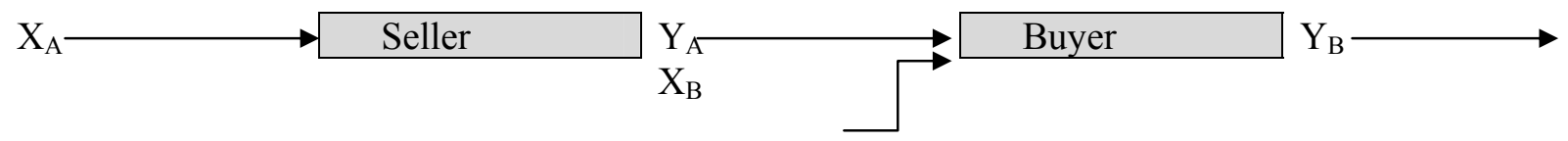

Fig. 1. Seller-Buyer Supply Chain

In this approach, the seller-buyer interaction is considered as a two-stage non-cooperative game with the seller as the leader and the buyer as the follower. First, we use the CCR model to evaluate the efficiency of the seller as the leader:

$$
E_{A A}^{*}=\max \sum_{r=1}^{s} u_{r}^{A} y_{r k}^{A}
$$

subject to

$$
\begin{array}{ll}
\sum_{i=1}^{m} v_{i}^{A} x_{i j}^{A}-\sum_{r=1}^{s} u_{r}^{A} y_{r j}^{A} \geq 0 & j=1, \ldots, n \\
\sum_{i=1}^{m} v_{i}^{A} x_{i j}^{A}=1 & \\
u_{r}^{A}, v_{i}^{A} \geq 0 & r=1, \ldots, s, \quad i=1, \ldots, m .
\end{array}
$$


Let $E_{A A}^{*}$ be the seller's efficiency, then we use the following model to evaluate the buyer's efficiency:

$$
\max \sum_{r=1}^{s} u_{r}^{B} y_{r k}^{B}
$$

subject to

$$
\begin{gathered}
d \times \sum_{r=1}^{s} u_{r}^{A} y_{r j}^{A}+\sum_{i=1}^{m} v_{i}^{B} x_{i j}^{B}-\sum_{r=1}^{s} u_{r}^{B} y_{r k}^{B} \geq 0 \\
d \times \sum_{r=1}^{s} u_{r}^{A} y_{r j}^{A}+\sum_{i=1}^{m} v_{i}^{B} x_{i j}^{B}=1 \\
\sum_{r=1}^{s} u_{r}^{A} y_{r k}^{A}=E_{A A}^{*} \\
\sum_{r=1}^{m} v_{r}^{A} x_{r j}^{A}-\sum_{i=1}^{s} u_{i}^{A} y_{i j}^{A} \geq 0 \\
\sum_{r=1}^{s} v_{r}^{A} x_{r k}^{A}=1 \\
u_{r}^{A}, v_{i}^{A}, u_{r}^{B}, v_{i}^{B}, d \geq 0
\end{gathered}
$$

$$
j=1, \cdots, n
$$

So $d \times \sum_{r=1}^{s} u_{r}^{A} y_{r o}^{A}+\sum_{i=1}^{m} v_{i}^{B} x_{i o}^{B}=1$ and $\sum_{r=1}^{S} u_{r}^{A} y_{r o}^{A}=E_{A A}^{*} ;$ thus, we have $0 \leq d<$ $\frac{1}{\sum_{r=1}^{S} u_{r}^{A} y_{r o}^{A}}=\frac{1}{E_{A A}^{*}}$. We then start to decrease $d$ according to $d_{p}=\frac{1}{E_{A A}^{*}}-\varepsilon \times p$ for each step $p$, where $\varepsilon$ is a small positive number. We solve each linear program of model (5) corresponding to $d_{p}$ and denotes the optimal objective value as $E_{A B}^{*}\left(d_{p}\right)$. Let $E_{A B}^{*}=\max E_{A B}^{*}\left(d_{p}\right)$. The efficiency of the supply chain can then be defined as follows,

$e_{A B}^{*}=\frac{1}{2}\left(E_{\mathrm{AA}}^{*}+E_{A B}^{*}\right)$

Similarly, one can develop a procedure for the situation when the buyer is the leader and the seller the follower. We first evaluate the efficiency of the buyer using the standard CCR model:

$$
E_{B B}^{*}=\max \frac{\sum_{r=1}^{s} u_{r}^{B} y_{r k}^{B}}{\sum_{r=1}^{s} u_{r}^{A} y_{r k}^{A}+\sum_{i=1}^{m} v_{i}^{B} x_{i k}^{B}}
$$

subject to

$$
\begin{array}{lc}
\frac{\sum_{r=1}^{s} u_{r}^{B} y_{r k}^{B}}{\sum_{r=1}^{s} u_{r}^{A} y_{r k}^{A}+\sum_{i=1}^{m} v_{i}^{B} x_{i k}^{B}} \leq 1 & j=1, \cdots, n \\
u_{r}^{A}, u_{r}^{B}, v_{i}^{B} \geq 0 & r=1, \ldots, s, \quad i=1, \ldots, m
\end{array}
$$

To obtain the seller's efficiency given that the buyer's efficiency is equal to $E_{B B}^{*}$, we solve the following model: 


$$
E_{B A}^{*}=\max \frac{U \times \sum_{r=1}^{s} u_{r}^{A} y_{r k}^{A}}{\sum_{i=1}^{m} v_{i}^{A} x_{i k}^{A}}
$$

subject to

$$
\begin{aligned}
& \frac{U \times \sum_{r=1}^{s} u_{r}^{A} y_{r k}^{A}}{\sum_{i=1}^{m} v_{i}^{A} x_{i k}^{A}} \leq 1 \\
& \sum_{r=1}^{s} u_{r}^{B} y_{r k}^{B}=E_{B B}^{*} \\
& \sum_{r=1}^{s} u_{r}^{A} y_{r j}^{A}+\sum_{i=1}^{m} v_{i}^{B} x_{i j}^{B}-\sum_{r=1}^{s} u_{r}^{B} y_{r k}^{B} \geq 0 \\
& \sum_{r=1}^{s} u_{r}^{A} y_{r j}^{A}+\sum_{i=1}^{m} v_{i}^{B} x_{i j}^{B}=1 \\
& u_{r}^{A}, v_{i}^{A}, u_{r}^{B}, v_{i}^{B}, U \geq 0
\end{aligned}
$$

The model (7), $U$ can be treated as a parameter, and this model can be solved as a parametric linear program. The efficiency of the supply chain can be defined as $e_{B A}^{*}=\frac{1}{2}\left(E_{B A}^{*}+E_{B B}^{*}\right)$

\subsection{Cooperative models}

In this section, we consider the case where all the members have the same degree of power to influence the supply chain system. This DEA model seeks to maximize efficiency all members, subject to a condition that the weights on the intermediate measures must be equal. Network DEA is a suitable approach for measuring efficiency of supply chain at cooperative model.

\subsubsection{Network DEA}

Systems with more than one process connected with each other are networks. To measure the efficiency of a network system, a network DEA model is needed. The proposed DEA is different from the conventional DEA model where the network DEA model does not have a standard form. It depends on the structure of the network in question.

\subsubsection{The product of members' efficiency (PE) in network DEA}

We can define the overall efficiency of supply chain as the product of individual efficiencies of members, subject to a condition that the weights on the intermediate measures must be equal;

Consider $n$ supply chains, where each has $h$ internal members. We suppose that $x_{i j}^{t} \in \mathcal{R}_{+}^{m_{t}}$ and $y_{r j}^{t} \in \mathcal{R}_{+}^{s_{t}}(t=1,2 \ldots, h)$ are inputs and outputs in the $t^{t h}$ member of supply chain $\mathrm{j}(\mathrm{j}=1, \ldots, \mathrm{n})$, respectively, and $z_{j}^{(t, f)}$ is the intermediate outputs which are emitted from member $t$ to member $f$, $(t, f) \in L$ (L is the number of intermediate outputs ).

We sort $h$ internal members of each supply chain as below: member 1 is a member that can transmit some of its own outputs to other members and remainder outputs are exited from system as direct outputs. In other words, its output is divided to two portions, $y_{r j}^{1}$ and $z_{j}^{(1, f)},(f=2, \ldots, h)$ which $y_{r j}^{1}$ 
output is exited from system directly and $z_{j}^{(1, f)}(f=2, \ldots, h)$ is sent to member $f$ as an input. The input of member 1 is provided by input resource directly. Member 2 of the system is a member that can transmit some portion of its own product to members $f=3, \ldots, h$ and exit the reminder directly from the system as output. In other words, its output is divided to $y_{r j}^{2}$ and $z_{j}^{(2, f)},(f=3, \ldots, h)$ which the former is gone out the system directly and the latter is used in member $f$ as an input. This member gains its input from input resource directly and also can gain some portion of its input from the output of member 1 . Therefore, we can say that the input of this member is made of two portions; $x_{i j}^{2}$ that is provided by main sources and $z_{j}^{(1,2)}$ which is a portions of output of member 1 . In this manner; the membership $t$ is a member that can transmit some portion of its outputs to member $t+1, \ldots, h$ and exit the reminder from system directly. It means that, its output is divided to $y_{r j}^{t}$ and $z_{j}^{(t, f)},(f=t+1, \ldots, h)$ which $y_{r j}^{t}$ is gone out directly from system and $z_{j}^{(t, f)}$ can be sent to members $(f=t+1, \ldots, h)$ as an input. This member can receive its own input directly from input resource and some portion of its input can be provided by members $1, \ldots, t-1$. It means that the input of this member are $x_{i j}^{t}$, which are provided directly by input resources, and $z_{j}^{(f, t)}$ which is a portion of output of members $\mathrm{f}=1, \ldots, \mathrm{t}-1$. According to this, We are able to measure the efficiency of supply chain $\mathrm{k}(k \in 1, \ldots, n)$ according model 8 .

In this case, after linearization we have the following model:

$$
\begin{array}{ll}
E_{k}^{*}=\max \sum_{t=1}^{h} \sum_{r=1}^{s} u_{r}^{t} y_{r k}^{t} & \\
\text { subject to } & \\
\sum_{t=1}^{h} \sum_{i=1}^{m} v_{i}^{t} x_{i k}^{t}=1 & j=1, \ldots, n \\
\sum_{r=1}^{s} u_{r} y_{r j}+\sum_{i=1}^{m} v_{i} x_{i j} \leq 0 & t=1, \ldots, h, j=1, \ldots, n \\
\sum_{r=1}^{s} u_{r}^{t} y_{r j}^{t}+\sum_{f=t+1}^{h} w(t, f) z_{j}^{(t, f)}-\sum_{i=1}^{m} v_{i}^{t} x_{i j}^{t}-\sum_{f=1}^{t-1} w(f, t) z_{j}^{(f, t)} \leq 0 & \\
u_{t}, u_{r}, w_{(t, f)}, w_{(f, t)}, v_{i} \geq 0 & j=1, \ldots, n, \ldots, f \neq t, \\
& f, t=1, \ldots, h, f \neq t,
\end{array}
$$

Let $\left(\mathrm{u}_{\mathrm{t}}^{*}, \mathrm{v}_{\mathrm{t}}^{*}, \mathrm{w}_{(\mathrm{t}, \mathrm{f})}^{*}, \mathrm{w}_{(\mathrm{f}, \mathrm{t})}^{*}\right)$ be the optimal solution of model (8), efficiency of member $t$ in supply chain $k$ is:

$$
\mathrm{E}_{\mathrm{k}}^{\mathrm{t}}=\frac{u_{t}^{*} \mathrm{y}_{\mathrm{k}}^{\mathrm{t}}+\sum_{\mathrm{f}=\mathrm{t}+1}^{\mathrm{h}} \mathrm{w}_{(\mathrm{t}, \mathrm{f})}^{*} \mathrm{z}_{\mathrm{j}}^{(\mathrm{t}, \mathrm{f})}}{\mathrm{v}_{\mathrm{t}}^{*} \mathrm{x}_{\mathrm{k}}^{\mathrm{t}}+\sum_{\mathrm{f}=1}^{\mathrm{t}-1} \mathrm{w}_{(\mathrm{f}, \mathrm{t})_{\mathrm{j}}^{*}}^{\mathrm{f}, \mathrm{t})}}
$$

\subsubsection{The weighted sum of members' efficiency (WSE) in Network DEA}

Instead of defining the overall efficiency of supply chain as the product of the individual efficiencies of members, we can compute the overall efficiency as the weighted sum of the efficiencies of members. The weight of member $t$, in evaluation, is $\mu_{t}(\mathrm{t}=1,2, \ldots, \mathrm{h})$, which user-specified weights satisfying $\sum_{t=1}^{\mathrm{h}} \mu_{\mathrm{t}}=1$. These weights are not decision variables, but rather are functions of decision variables, reflecting the relative importance or contributions of the performances of each member in supply chain to its overall performance. The efficiency of supply chain is $\theta=\sum_{t=1}^{h} \mu_{t} \theta_{t}$, which $\theta_{t}$ is efficiency of member $t$ : 
$\theta_{t}=\frac{u_{t} y_{j}^{t}+w_{(t, k)} z_{j}^{(t, k)}}{w_{(f, t)} z_{j}^{(f, t)}+v_{t} x_{j}^{t}}$

where $\mathrm{z}_{\mathrm{j}}^{(\mathrm{f}, \mathrm{t})}$ is intermediate outputs from member $f$ to member $t$.

$$
\begin{aligned}
& \theta_{k}^{*}=\max \sum_{t=1}^{h}\left(u_{t} y_{k}^{t}+w_{(t, k)} z_{k}^{(t, k)}\right) \\
& \text { subject to } \\
& \qquad \sum_{t=1}^{h} w_{(f, t)} z_{k}^{(f, t)}+v_{t} x_{k}^{t}=1 \\
& \quad u_{t} y_{j}^{t}+w_{(f, t)} z_{k}^{(f, t)}+v_{t} x_{k}^{t}=1 \\
& \quad u_{t} y_{j}^{t}+w_{(t, k)} z_{k}^{(t, k)}-w_{(f, t)} z_{k}^{(f, t)}-v_{t} x_{j}^{t} \leq 0 \\
& \quad u_{t}, w_{(i, j)}, v_{t} \geq 0 \quad t=1, \ldots, h \quad t=1, \ldots, h, j=1, \ldots, n
\end{aligned}
$$

By setting $\mu_{t}=\frac{w_{(f, t)} z_{j}^{(f, t)}+v_{t} x_{j}^{t}}{\sum_{t=1}^{h}\left(w_{(f, t)} z_{j}^{(f, t)}+v_{t} x_{j}^{t}\right)} \quad$ which it shows the relative sizes of the members, so $\theta=$ $\sum_{\mathrm{t}=1}^{\mathrm{h}} \mu_{\mathrm{t}} \theta_{\mathrm{t}}$, then the objective function of (10) can then be rewritten as

$$
\theta^{*}=\sum_{t=1}^{h}\left(\frac{u_{t} y_{j}^{t}+w_{(t, k)} z_{j}^{(t, k)}}{w_{(f, t)} z_{j}^{(f, t)}+v_{t} x_{j}^{t}}\right)
$$

Theorem. Let $E_{k}^{*}$ and $\theta_{k}^{*}$ be the overall efficiencies of $D M U_{k}$ under CCR assumption determined by network DEA models of (8) and (10) respectively. Then $E_{k}^{*} \leq \theta_{k}^{*}$.

\section{Numerical Examples}

In example 1, we compare the efficiency scores for the cooperative and non-cooperative models.

Example 2 indicates the overall efficiency of supply chain as the product of individual efficiencies of members versus the overall efficiency as the weighted sum of the individual efficiencies of members.

Example 1. Consider seller-buyer supply chains where the seller has two inputs (shipping cost and labor; $x_{1}^{A}, x_{2}^{A}$ ) and two outputs (buyer's fill rate and number of product shipped; $y_{1}^{A}, y_{2}^{A}$ ), and the buyer has another input (labor; $x^{B}$ ) and one output (profit; $y^{B}$ ). Data of inputs and outputs of five supply chains (DMUs) are given in Table 1.

\section{Table 1}

Inputs and outputs of members of supply chains

\begin{tabular}{lllllll}
\hline DMU & \multicolumn{1}{c}{$x_{1}^{A}$} & $x_{2}^{A}$ & $y_{1}^{A}$ & $y_{2}^{A}$ & $x^{B}$ & $y^{B}$ \\
\hline 1 & 8 & 50 & $20 \%$ & 10 & 8 & 100 \\
2 & 10 & 18 & $10 \%$ & 15 & 10 & 70 \\
3 & 15 & 30 & $10 \%$ & 20 & 8 & 95 \\
4 & 8 & 25 & $20 \%$ & 25 & 10 & 80 \\
5 & 10 & 40 & $15 \%$ & 20 & 15 & 85 \\
\hline
\end{tabular}

Table 2 shows the efficiency scores obtained from the supply chain efficiency models. Supply chain is efficient if and only if it's all members are efficient. As can be seen in Table 2, if all members of supply chain are efficient then supply chain will be efficient. The first supply chain is fully efficient in the above example. The efficiency in PE network model is always less than or equal efficiency of non-cooperative models. The supply chain 5 has the least efficiency among the other chains in PE network model and in both non-cooperative models. Therefore, there is a suitable accordance between PE network model and the non-cooperative models. The calculation in network models is less than non-cooperative models. 
Table 2

Efficiency of supply chain in models non-cooperative and model (8)

\begin{tabular}{cccccccccc}
\hline Supply chain & $E_{A A}$ & $E_{A B}$ & $e_{A B}$ & $E_{B B}$ & $E_{B A}$ & $e_{B A}$ & $\begin{array}{c}E_{A} \text { in model } \\
(8)\end{array}$ & $\begin{array}{c}E_{B} \text { in model } \\
(8)\end{array}$ & $\begin{array}{c}\text { Overall efficiency in } \\
\text { model (8) }\end{array}$ \\
\hline 1 & 1 & 1 & 1 & 1 & 1 & 1 & 1 & 1 & 1 \\
2 & 0.83 & 0.56 & 0.7 & 0.88 & 0.77 & 0.82 & 0.77 & 0.88 & 0.67 \\
3 & 0.67 & 0.95 & 0.81 & 1 & 0.55 & 0.77 & 0.1 & 0.95 & 0.95 \\
4 & 1 & 0.65 & 0.83 & 0.65 & 1 & 0.83 & 1 & 0.65 & 0.65 \\
5 & 0.64 & 0.45 & 0.55 & 0.76 & 0.62 & 0.69 & 0.60 & 0.35 & 0.47 \\
\hline
\end{tabular}

Consider $n$ supply chains, which each of them have $h$ internal members, the number of constraint is $h n+1$. In supply chain with two members, the number of constraints in network model (8) is $2 n+1$, while cooperative model need to solve two models that the first model has $n+1$ constraints and the second model has $2 n+1$ constraints, also the second model is parametric, so we should solve several model of the second model; therefore the stage for solving non-cooperative model is very more than network model.

Example 2. Consider supply chains as Fig. 2. These supply chains include two members. The member 1 has three inputs and three outputs, while the member 2 has four inputs (three inputs are outputs of the member 1 and one directly input) and two outputs.

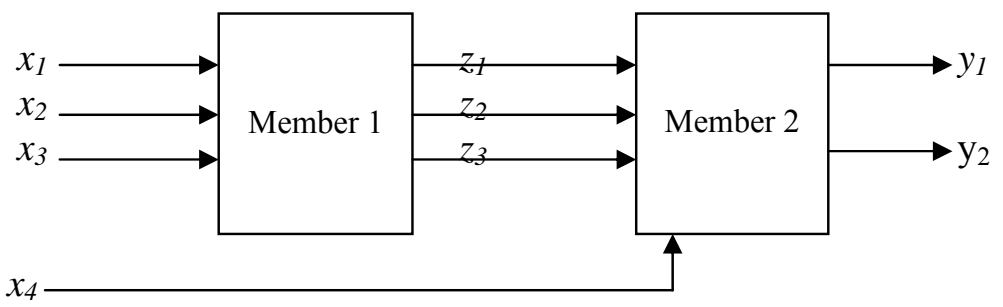

Fig. 2. A sample of supply chain in example 2

Data of members inputs and outputs of10 supply chain indicated in Table 3. Efficiency of supply chains and their members in two models of cooperative, using network DEA are shown in Table 4. The results of the example are according to above theorem, the overall efficiency of PE model is less than or equal the overall efficiency in the WSE model. If a supply chain is efficient in PE model, it is efficient in WSE model; also inefficient supply chain in the WSE model is inefficient in PE model.

Table 3

Inputs and outputs of members of supply chains of example 2

\begin{tabular}{cccccccccc}
\hline Supply chain & $x_{1}$ & $x_{2}$ & $x_{3}$ & $z_{1}$ & $z_{2}$ & $z_{3}$ & $x_{4}$ & $y_{1}$ & $y_{2}$ \\
\hline 1 & 9 & 50 & 1 & 20 & 10 & 5 & 8 & 100 & 25 \\
2 & 10 & 18 & 10 & 10 & 15 & 7 & 10 & 70 & 20 \\
3 & 9 & 30 & 3 & 8 & 20 & 2 & 8 & 96 & 30 \\
4 & 8 & 25 & 1 & 20 & 20 & 10 & 10 & 80 & 20 \\
5 & 10 & 40 & 5 & 15 & 20 & 5 & 15 & 85 & 15 \\
6 & 7 & 35 & 2 & 35 & 10 & 5 & 5 & 90 & 35 \\
7 & 7 & 30 & 3 & 10 & 25 & 8 & 10 & 100 & 30 \\
8 & 12 & 40 & 4 & 20 & 25 & 4 & 8 & 120 & 10 \\
9 & 9 & 25 & 2 & 10 & 10 & 5 & 15 & 110 & 15 \\
10 & 10 & 50 & 1 & 20 & 15 & 9 & 10 & 80 & 20 \\
\hline
\end{tabular}

In both model, the supply chain 6 is efficient, and supply chain 5 is the most inefficient. According to Table 3, inputs of supply chain 7 are less than inputs of supply chain 8 and outputs of supply chain 7 are more than outputs of supply chain, so efficiency of 7 should be more than efficiency of 8; this case occur in PE model. 
Table 4

Efficiency of supply chain in model (8) and (10)

\begin{tabular}{|c|c|c|c|c|c|c|}
\hline DMU & $\begin{array}{l}\text { Overall efficiency } \\
\text { in model ( } 8)\end{array}$ & $\begin{array}{r}E_{A} \text { in } \\
\operatorname{model}(8)\end{array}$ & $\begin{array}{r}E_{B} \text { in } \\
\text { model (8) }\end{array}$ & $\begin{array}{l}\text { Overall efficiency } \\
\text { in model (10) }\end{array}$ & $\begin{array}{c}E_{A} \text { in } \\
\text { model }(10)\end{array}$ & $\begin{array}{r}E_{B} \text { in } \\
\text { model (10) }\end{array}$ \\
\hline 1 & 0.9 & 0.76 & 1 & 0.92 & 0.76 & 1 \\
\hline 2 & 0.73 & 0.9 & 0.8 & 0.86 & 0.9 & 0.8 \\
\hline 3 & 0.83 & 0.47 & 1 & 0.86 & 0.59 & 1 \\
\hline 4 & 0.63 & 1 & 0.63 & 0.77 & 1 & 0.55 \\
\hline 5 & 0.45 & 0.13 & 0.54 & 0.62 & 0.68 & 0.57 \\
\hline 6 & 1 & 1 & 1 & 1 & 1 & 1 \\
\hline 7 & 0.82 & 1 & 0.82 & 0.9 & 1 & 0.81 \\
\hline 8 & 0.78 & 0.7 & 0.91 & 0.93 & 0.67 & 1 \\
\hline 9 & 0.72 & 0.5 & 1 & 0.78 & 0.5 & 1 \\
\hline 10 & 0.63 & 0.88 & 0.66 & 0.75 & 0.84 & 0.65 \\
\hline
\end{tabular}

\section{Conclusion}

This paper presented several DEA models for evaluating the overall performance of a supply chain and its members. The non-cooperative model is modeled as a leader-follower structure, and the cooperative models try to maximize the joint efficiency of the members. Network DEA is a suitable approach for measuring efficiency of supply chain at cooperative model. In the leader-follower (or non-cooperative) structure, the leader is first evaluated using the standard DEA model, and then the follower is evaluated by a DEA-based model which incorporates the DEA efficiency information for the leader. The extension of non-cooperative models for supply chains with more than two members is very complex ratio to Cooperative models. The Cooperative models, especially in network models are easily used for any number of members in the supply chain. The proposed model, based on network DEA model, calculates overall efficiency of supply chain as product of efficiency of supply chain members; also the relationship between members are not limited to two members, and each member can be connected with other members. In this paper, the network models are divided to two kinds; in the first kind, overall efficiency of supply chain is calculated as the product of efficiencies of members and the second kind compute the overall efficiency as the weighted sum of the efficiencies of members. The number of constraint in evaluation of $n$ supply chain, which each of them has $\mathrm{h}$ internal members using network DEA is $\mathrm{hn}+1$. The number of variable in the second kind is more than variable in the first kind. While non-cooperative models are parametric and the optimization need to solve several linear programming. Therefore, the proposed method reduced computations and it directly evaluate the performance of the supply chain as well as its members, while considering the relationship between the members.

\section{References}

Chen Y., \& Zhu J. (2004). Measuring information technology's indirect impact on firm performance. Information Technology \& Management Journal, 5(2), 9-22.

Cook, W., Liang L., \& Zhu J. (2010). Measuring Performance of tow-stage network structures by DEA: A review and future perspective. Omega, 38, 423-430.

Fare, R., \& Grosskopf, S. (2000). Network DEA. Socio-Economic Planning Sciences, 34, 35-49.

Golany, B., \& Hackman, S. T., \& Passy, U. (2006). An efficiency measurement framework for multistage production systems. Annals of Operations Research, 145(1), 51-68.

Kao, C. (2009). Efficiency decomposition in network data envelopment analysis: A relational model. European Journal of Operational Research, 192, 949-962.

Liang L., Yang F., Cook W. D., \& Zhu J. (2006). DEA model for supply chain efficiency evaluation. Annals of Operations Research, 145, 35-49.

Seiford, L. M., \& Zhu J. (1999). Sensitivity and stability of the classification of returns to scale in data envelopment analysis. Journal of Productivity Analysis, 12, 55-75. 